\title{
Production of Cell Enclosing Silk Derivative Microsphere in Uniform Size Distribution Through Coaxial Microfluidic Device and Horseradish Crosslinking Reaction
}

\section{Elham Badali}

Kharazmi University

Mahshid Hosseini

Sharif University of Technology

Narges Mahmoodi

Tehran University of Medical Sciences

\section{Sajad Hassanzadeh}

Iran University of Medical Sciences (IUMS)

\section{Vajihe Taghdiri Nooshabadi}

Semnan University of Medical Science

\section{Arash Goodarzi}

Fasa University of Medical Sciences

\section{Maryam Jalessi}

Iran University of Medical Sciences (IUMS)

\section{Zohreh Arabpour}

Tehran University of Medical Sciences

Mehdi Khanmohammadi ( $\nabla$ Mehdi.khanmohammadi84@gmail.com )

Iran University of Medical Sciences (IUMS)

\section{Research Article}

Keywords: Cell-laden microparticle, Enzymatic reactions, Microfluidic devices, Silk fibroin, Spherical microtissue

Posted Date: October 6th, 2021

DOl: https://doi.org/10.21203/rs.3.rs-895554/v1

License: (9) This work is licensed under a Creative Commons Attribution 4.0 International License. Read Full License 


\section{Abstract}

\section{Background}

Silk fibroin (SF) as a natural polymer holds great potential in biomedical research because of its biocompatibility, easy processing, high toughness, and strength. However, slow gelation time has narrowed its applications, specifically in cell-laden microparticles that are versatile structures for tissue engineering due to their unique features. In addition, most crosslinking methods used to decrease gelation time did not occur in a mid-condition.

\section{Methods}

This study aimed to use modified SF with phenol conjugation to accelerate crosslinking mediated via horseradish peroxidase (HRP)/ hydrogen peroxide $\left(\mathrm{H}_{2} \mathrm{O}_{2}\right)$ in a co-flow high-throughput microfluidic device for the ultimate goal of cell-laden silk fibroin-phenol (SF-Ph) microparticles formation. The physical and biochemical properties of fabricated cell-laden SF-Ph were evaluated to reveal its potential for tissue engineering.

\section{Results}

The monodisperse microparticles in shape and size were formed in various diameters changing from 300 to $80 \mu \mathrm{m}$ by altering oil phase velocity from SF-Ph substrate. More than $90 \%$ cell viability and three times cells upregulation of mitochondrial activity of enclosed-cells in microparticles with $150 \pm 32 \mu \mathrm{m}$ diameters revealed that these structures were suitable subcultures produced through a mild process based on morphological and MTT assays. It was noticed that cells approximately cover the microparticles until the 15 th day.

\section{Conclusion}

Spherical micro-tissue formation in microparticles, resulting from cell growth promoted by cell-cell and cell-matrix interactions, adds significant weight to this method's applications.

\section{Introduction}

Silk fibroin (SF), the chief composition of silk obtained from fiber spinning during cocoons formation, has heavily attracted researchers because it is a high-quality natural protein $(1,2)$ with favorable features including ease to process, controllable biodegradation $(1,3)$, biocompatibility resulting in weak antigenic effects, and inflammatory response in-vivo (4-6). Hierarchical self-assembly of SF structure in nature is composed of amorphous and crystalline domains, imparting high toughness and strength to silk, leading to unique mechanical properties $(1,7)$. Owing to these, SF has found its way in many fields, including sustained drug delivery, biosensing, adhesives, wound repair, tissue engineering in several forms: nanofibers, membranes, scaffolds, hydrogels, microstructures $(1,4,8-10)$. The SF utilized in tissue engineering is mainly modified and crosslinked to form a three-dimensional (3D) shape (11). 
There are currently two main approaches for crosslinking SF, divided into physical and chemical crosslinking (1). In physical crosslinking, exertion of environmental stimulators such as electric field (12), shear action (13), use of surfactants (14), etc., could accelerate the transition of SF solution to $\beta$-sheet conformation. Although the physical crosslinking method is favorable in the term of operation, the noncovalent bonds diminish mechanical properties of the final structures compared to chemical crosslinking (1). Chemical crosslinking methods such as visible-light induction (15) or UV-light (16), enzymatic (7), schiff base (17) and, click chemistry (18) provide hydrogels with tunable properties, including porosity, swelling ratio, degradation rate, and mechanical properties as well as biochemical properties $(1,19)$. Among many ways of chemical crosslinking methods, enzymatic crosslinking reactions, using biological enzymes to catalyze crosslinking among SF side chains, occurs in an aqueous and mild condition, making it a great candidate for cell encapsulation $(1,7)$. One of the commonly used enzymes is HRP, activating intrinsic phenol groups of tyrosine on $\mathrm{SF}$ to dityrosine in the presence of $\mathrm{H}_{2} \mathrm{O}_{2}$ as an electron donor (20). Nevertheless, enzymatically crosslinked SF hydrogels are still relatively slow reactions, specifically in physiological media compared to water (21), which have made limitations for using this polymer in 3D bioprinting and cell microencapsulation by microfluidic devices, requiring a rapid gelation time $(22,23)$. To address this issue, 0 . Hasturk et al. (7) suggested modifying SF by introducing phenol moieties to increase available phenol groups in $\mathrm{HRP} / \mathrm{H}_{2} \mathrm{O}_{2}$-mediated reaction, which revealed a combination of SF and SF-Phenol enhanced gelation time, mechanical stability, and delayed biodegradation of the final hydrogel, resulting from an approximately mild reaction condition. The desired manageable properties of hydrogel obtained from enzymatical reaction possibly pave the way for using SF features to produce cell-laden microparticles that are attractive structures for different usages.

Cell-laden microparticles have broadly taken researchers' attention owing to mimicking natural extracellular matrix by a hydrated network and 3D microenvironment (24), large surface-to-volume of microparticles that facilitate efficient transfer of oxygen, nutrition, growth factor, therapeutic products, and metabolic wastes, cell-cell, and cell-matrix interactions $(25,26)$. These desired features broaden their applications in many fields, including stem cell differentiation (27), spherical microtissue formation (for disease modeling (28), drug testing (29), regenerative medicine (30), the building block for complex tissue construction by bioprinting (31)), cell delivery to the infarcted tissues with additional positive effects on enclosed cells by protecting them from immune response and harsh environment $(32,33)$. Therefore, many efforts have been made to generate microparticles. Among them, microfluidic devices provide worthy features that distinguish them for this purpose, including precise control over microparticle size distribution, composition, shape, and size (24). On this subject, several articles have been successfully fabricated microparticles from different phenol-substituted polymers such as alginate (34) and hyaluronic acid (35) through the $\mathrm{HPR} / \mathrm{H}_{2} \mathrm{O}_{2}$ mediated reaction with the high-throughput co-flow microfluidic device.

In this study, we used the same technique to probe the possibility of cell-laden SF-Ph microparticle fabrication. The produced cell-laden SF-Ph macroparticle with this method is reported for the first time in this study. The microparticles were formed through the emulsion process of the polymer precursor in the 
oil phase, which is well-controlled by a coaxial microfluidic flow and concentration adjustment. Then, they became polymerized in the rapid enzymatic reaction that HRP catalyzed the transfer of electrons from $\mathrm{H}_{2} \mathrm{O}_{2}$ supplied by the oil phase to conjugate available $\mathrm{Ph}$ groups to each other. Identical shape and size of microparticles, showing that we found out standard parameters including SF-Ph concentration, reactants, volumetric flow of solutions in a microfluidic device, and cellular density. The effect of microencapsulation on the recovered cell growth, the profile of enclosed cell proliferation, and the possibility of spherical microtissue were evaluated.

\section{Materials And Methods}

\subsection{Materials}

Bombyx mori silk cocoons were provided by the Iranian silkworm research center (Guilan, Iran). All materials used in this project were purchased from Sigma-Aldrich. Cells were cultured in Dulbecco's Modified Eagle Medium: Nutrient Mixture F-12 (DMEM/F-12) media supplemented, fetal bovine serum (FBS) $10 \%$, penicillin-streptomycin $100 \mu \mathrm{g} / \mathrm{mL}$, which were obtained from Gibco (USA). In this study, instead of stem cells, L929 mouse fibroblast cell was chosen as the model cell due to its good compatibility with hydrogels. The L929 cell was obtained from the National Cell Bank of Pasteur Institute of Iran.

\subsection{Silk fibroin (SF) extraction}

SF was extracted from Bombyx mori cocoons following some modification in the previously described method (7). The $10 \mathrm{~g}$ of cut $\mathrm{B}$. mori cocoons were degummed by boiling via $1 \mathrm{~L}$ of an aqueous medium containing $0.02 \mathrm{M} \mathrm{Na}_{2} \mathrm{CO}_{3}$ for 40 min to remove silk protein sericin; then, the fibers were entirely rinsed using deionized (DI) water. Specimens were soaked in DI water for $20 \mathrm{~min}$ at $80^{\circ} \mathrm{C}$ and rinsed with cold water forth times to further extraction of the glue-like sericin protein. They were allowed to dry overnight under a chemical hood. The prepared sample was dissolved in $9.3 \mathrm{M}$ lithium bromide ( $\mathrm{LiBr})$ at $60{ }^{\circ} \mathrm{C}$ for 4 $\mathrm{h}$, to a make $20 \%(\mathrm{w} / \mathrm{v}$ ) solution. The SF solution was dialyzed (MWCOs of $12 \mathrm{kDa}$ ) against DI water for three days, refreshing the DI water each 3-4 h. Insoluble components were isolated from the dialyzed solution by centrifuge at $5000 \mathrm{rpm}$ for $15 \mathrm{~min}$ and finally lyophilized-dried. The final solution was kept at room temperature for subsequent steps. Weighing the SF before and after freeze-drying in a known volume indicated the concentration of SF.

\subsection{Synthesis of silk fibroin-phenol (SF-Ph)}

SF-Ph was synthesized via carbodiimide-mediated reaction as described elsewhere (7). Briefly, $10 \mathrm{~g} \mathrm{SF}$ was dissolved in $30 \mathrm{mM}$ 2-(N-morpholino) ethanesulfonic acid (MES) buffer at $40 \mathrm{~g} / \mathrm{L}$. To this solution, tyramine hydrochloride, water-soluble carbodiimide hydrochloride (WSCD), and $n$ - 
hydroxysuccinimide (NHS) were added at 2.7, 2.1, and $2.9 \mathrm{~g} / \mathrm{L}$. The mixture was stirred using a magnetic bar for $24 \mathrm{~h}$ at room temperature. The remaining chemicals in the resultant solution were removed by dialysis using a $12 \mathrm{kDa}$ dialysis membrane against the surplus amount of distilled water for three days, whereas distilled water refresh every 3-4 h. The final SF-Ph solution was maintained at $4{ }^{\circ} \mathrm{C}$ for more usages.

\subsection{SF-Ph characterization}

UV-visible spectrophotometer and proton nuclear magnetic resonance ( ${ }^{1} \mathrm{H}$ NMR) spectroscopy were performed to figure out the content of phenol adhering to polymers $(34,36)$ and further confirm the conjugation of phenol moieties on SF (7). In more detail about UV visible spectrophotometer, the standard curve was plotted to determine the Ph contents of synthetic SF-Ph resulting from the absorbance of known tyramine hydrochloride concentration at $275 \mathrm{~nm}$ (34). It is worth mentioning that in the ${ }^{1} \mathrm{H}$ NMR test, SF and SF-Ph were diluted 1-fold with deuterium oxide $\left(\mathrm{D}_{2} \mathrm{O}\right)(37)$.

\subsection{Process of cell-enclosing microparticles}

SF-Ph-based microparticles were fabricated using a designed microfluidic co-axial double-orifice spinneret, which was similarly applied for fabrication microparticles from other polymers, as shown in Fig. $1(34,35)$. For this, the inner solution containing SF-Ph at $4 \%(w / v), L 929$ at $4 \times 10^{6} \mathrm{cells} / \mathrm{mL}$, and 40 unit/mL HRP were extruded through the inner channel of the microfluidic device in $180 \mu \mathrm{m}$ diameter at 70 $\mu \mathrm{L} /$ min. The immiscible liquid paraffin containing lecithin and saturated $\mathrm{H}_{2} \mathrm{O}_{2}$ flowed into the outer channel of the microfluidic device with $600 \mu \mathrm{m}$ diameter at $3 \mathrm{~mL} / \mathrm{min}$. This liquid paraffin containing $\mathrm{H}_{2} \mathrm{O}_{2}$ and $3 \%(\mathrm{w} / \mathrm{w}$ ) lecithin was prepared by stirring $60 \mathrm{~mL}$ of liquid paraffin and $600 \mu \mathrm{LL}$ of aqueous $\mathrm{H}_{2} \mathrm{O}_{2}$ solution ( $12 \mathrm{M}$ ) using a homogenizer for $10 \mathrm{~min}$ in moderate speed. The resultant emulsion was centrifuged at $3000 \mathrm{rpm}$ to isolate the surplus $\mathrm{H}_{2} \mathrm{O}_{2}$ solution from liquid paraffin and then mixed with a 3fold volume of liquid paraffin containing $4 \%(w / w)$ lecithin. The microparticles were formed through droplet formation at the intersection of two flows in the vicinity of the microfluidic device when SF-Ph and liquid paraffin solutions were dispersed phase and continuous phase, respectively. Effects of flow rates of aqueous SF-Ph solution and liquid paraffin flow as well as concentrations of SF-Ph on the diameter of droplets were monitored utilizing syringe pumps and prepared concentrated SF-Ph solution, respectively. The droplets of SF-Ph solution in the liquid paraffin were collected in a $50 \mathrm{~mL}$ Corning ${ }^{\circledR}$ tube positioned at the tip of the outer channel. The droplets were collected through centrifugation at $1000 \mathrm{rpm}$ for $1 \mathrm{~min}$ to allow the collection of droplets and rinsed using calcium-free Krebs Ringer HEPES-buffered saline (CF$\mathrm{KRH}$ buffer). The collected droplets were transferred in a $15 \mathrm{~mL}$ Corning ${ }^{\circledR}$ tube with excess CF-KRH buffer to wash the remaining liquid paraffin. Then, the sample was centrifuged at $1500 \mathrm{rpm}$ for $4 \mathrm{~min}$. The collected SF-Ph microparticles were washed twice with $\mathrm{KRH}$ to completely remove the liquid paraffin. The mean diameter of the resultant SF-based microparticles was determined based on measurements of 
more than 100 specimens using an optical microscope. After removing paraffin from the cell-enclosing microparticles by successive centrifuges, those were placed in a culture medium.

\subsection{Enclosing effects on cells}

The assessment of possible adverse enclosing effects on cell growth was accomplished by MTT (3-(4,5dimethylthiazol-2-yl)-2,5-diphenyltetrazolium bromide) assay at different time point intervals, including just and 12 days after microparticles preparation. Cell-enclosing microparticles were firstly degraded using collagenase type II at $200 \mathrm{unit} / \mathrm{mL}$ concentration within $1 \mathrm{~h}$ incubation. Then the viability of the harvested cells was determined by trypan blue exclusion dye assay. To evaluate possible damages requiring time to suppress cellular growth, harvested cells from microparticles were seeded at a density of $1 \times 10^{4}$ cells/well in a 48-well plate to examine cell morphology and viability. The MTT solution at a final concentration of $0.5 \mathrm{mg} / \mathrm{mL}$ was poured into seeded cells in each well, incubated for $4 \mathrm{~h}$ in physiological condition. The dimethyl sulfoxide (DMSO) was replaced with MTT solution to dissolve formazan, resulting from the reduction of tetrazolium dye MTT by NAD (P)H enzyme. Finally, the absorbance of DMSO, a viable cell indicator, at $570 \mathrm{~nm}$ was read by ELISA Microplate Readers $(35,38)$.

\subsection{Enclosed cells activity assay}

To determine the growth profile of encapsulated cells in microparticles on different days, the MTT assay was performed similar to the previously mentioned method. Briefly, MTT solution was added to 100-200 counted microparticles containing cells. After changing MTT solution with DMSO, the colour intensity of DMSO was measured by ELISA Microplate Readers at $570 \mathrm{~nm}$ (38).

\subsection{Spherical microtissue fabrication}

To fabricate microtissues, cell-laden microparticles, which were produced from SF-Ph solution containing L929 cells, were incubated for 15 days, allowing sufficient time for cell growth to occupy microstructures based on microscopic images. Then, microparticles were immersed in a culture medium supplemented with 200 unit/mg collagenase type II for one hour to digest SF-Ph microparticles. The forms of naked spherical microtissue were observed via a microscopic image.

\section{Results And Discussions}

Development The development of biocompatible cellular constructs in non-diffusion limit size can provide suitable physicochemical and biological properties to support cell viability and functions, including cell adhesion, proliferation and differentiation $(39,40)$. The SF is one of the valuable and applicable natural polymers for its high strength $(1,7)$, good biological compatibility, and low immunogenicity that resulted in a wide application for various biomedical purposes (4-6). It can be 
physically and chemically modified to amend the biophysical and biochemical properties of SF-based scaffolds and constructs for tissue engineering and drug delivery purposes $(1,10)$. SF-based constructs can be fabricated through different techniques and shapes of architectures such as films, sponges, nano/micro-particles, fibers/tubes, bulk hydrogel, and conduit $(1,8,10)$.

We prepared the scaffolds using extracted SF. The cellular vehicle should mimic an extracellular matrix and possess a 3D structure $(39,41)$. We applied modified SF with phenol moieties to fabricate the new composite scaffolds with good microstructure and suitable biocompatibility and mechanical properties for various biomedical applications, including tissue engineering and drug or cellular delivery. The spherical-shaped micro vehicles were obtained through conjugation of $\mathrm{Ph}$ moieties to the backbone of SF and in following using the microfluidic technique, which performed HRP-catalysed crosslinking reaction during particle fabrication.

\subsection{SF-Ph synthesis and characterization}

As depicted in Fig. 2A, SF polymer was modified by conjugation of $\mathrm{Ph}$ moieties deriving from tyramine hydrochloride in the presence of ethyl(dimethylaminopropyl) carbodiimide (EDC) and NHS (7). As shown in Fig. $2,{ }^{1} \mathrm{H}$-NMR test elucidated peaks from 6.8 to $7.2 \mathrm{ppm}$, referring to the aromatic protons of the phenolic group based on reports $(37,42)$. In the mentioned region, striking differences were noticed between SF and SF-Ph peaks, prove successful synthesis. The Ph content introduced to modified SF was measured by UV visible spectrophotometer, which was $1.24 \times 10^{-4} \mathrm{~mol}-\mathrm{Ph} / \mathrm{g}$-SF-Ph. Since the reported Ph content was close to related works $(35,43)$, which have been utilized other polymers to fabricate microparticles, this synthesized polymer would also be suitable for that.

\subsection{Preparation of SF-Ph microparticles}

We evaluated the possibility of SF-Ph microparticle formation by the designed coaxial microfluidic device and enzyme-mediated crosslinking reaction using HRP in the presence of $\mathrm{H}_{2} \mathrm{O}_{2}$ as an electron donor. As microphotographs show, microparticles were successfully produced via the HRP-mediated enzymatic reaction, which was in agreement with other reports related to microparticles fabrication with other polymers which were modified with $\mathrm{Ph}$ moieties and used for enzymatic reactions such as hyaluronic acid (HA), alginate, carboxymethyl cellulose, and amylopectin, and gelatin (35). As here liquid paraffin solution as a continuous phase meets the SF-Ph solution as a dispersed phase, shear stress causes the SF-Ph solution to pinch-off and form droplets. In fact, shear stress is caused by a reduction in the size of the dispersed phase channel at the orifice, at which hydrodynamic flow-focusing occurs. For further study, the microparticles size dependency was investigated by changing the flow rate of paraffin at the constant SF-Ph solution flow rate of $75 \mu \mathrm{L} / \mathrm{min}$ with the optical microscopic images, in which SF concentration was $4 \%(w / v)$ in the final solution. Image $J$ was used to analyze resultant images, showing that paraffin velocity changes from 0.75 to $4.2 \mathrm{~mL} / \mathrm{min}$ gave rise to changes in microparticles diameters 
approximately from 300 to $80 \mu \mathrm{m}$, as illustrated in Fig. 3 A and B. It was evident that an increase in liquid paraffin solution flow rate decreased microparticles diameter, as was proved in various reports (35), confirming microparticles diameter dependency on liquid paraffin flow rate as a volumetric-driven flow force. The uniformity in size distribution and high cell viability are critical parameters for cell encapsulation, and also, there is size diffusion limit for cellular constructs. The size below $200 \mu \mathrm{m}$ is highly recommended for cellular vehicles $(40,44)$. As shown in Fig. 3 C, the mean diameter of microparticles was considered about $150 \mu \mathrm{m}$ for the following experiments to minimize the formation of necrotic regions in the microparticles' canter (44). Microphotograph and narrow size distribution for fabricated microparticles confirm that specimens were in uniform size and spheroid shape. These properties give us this opportunity to utilized SF based microparticles for cell or drug delivery in specified density or concentration, respectively. The average size of microparticles follows a Poisson-like distribution and can be finely controlled by flow velocity. We did not observe any structural instability, including coalescence of SF-Ph hydrogel in micron size, which means proper concentration of SF-Ph polymer, reactants, volumetric flow of solutions in a microfluidic device, and cellular density. It is reported that these parameters can cause breakage of particles as well as their coalesce into a bigger size or nonsize uniformity such as pear, oval, and spindle shape vehicles even if the surfactant concentration in the collection solution be quite high (45) .

\subsection{Cellular behaviour}

The probable side effects of enclosing cells can be associated to lack of culture medium which means lack of oxygenation and nutrition combined with shear stresses causes in the microencapsulation process, including mixing cells with viscus polymeric solution of SF-Ph, the existence of chemical reactants, and hydrogelation as well as shear stress through perfusion flow in microfluidic channels (35, 38). Cellular viability and activity were evaluated by both microscopic images and MTT assay at two-time points just and 12 days after cell enclosing (35). Morphologically, the spread up of cells in the initial hours of seeding, which were harvested from the encapsulation process and even after 12 days, were similar and did not find any difference among their morphologies and growth ability (Fig. 4A-F). Moreover, cellular growth shows harvested cells from encapsulation in different time-lapse (Fig. 4G) proliferated equally and mitochondrial activity of cells which indicate lack of any cytotoxicity of encapsulation process and extension time of incubation for encapsulated cells.

\subsection{Enclosed cells activity assay}

The viability and proliferation of enclosed cells are the deterministic factors in considering a suitable cell carrier (24). Hence, cell growth in microparticles was evaluated by microscopic images and MTT assay on different days after microencapsulation. Until the 15th day, embedded L929 cells vividly proliferated and occupied all regions of microparticles, illustrated in Fig. 5A-F. In addition, the quantitative analysis of cellular growth has proceeded through the MTT assay. Fig. 5. indicated that cell mitochondrial activity 
elevated until the 18 days of microencapsulation, which can be attested to denser microstructures (46). The cellular growth and proliferation in SF-Ph microparticles would be due to the existence of cell interactive moieties in SF structure, such as cell adhesive motifs $(10,47)$. Meanwhile, cellular growth on hydrogel surfaces for most polysaccharides and synthetic polymers hindered and obtained a low proliferation rate. Protein-based hydrogels such as gelatin, silk, and collagen could promote cellular growth even 3D cell culture system for encapsulated cells which microscopic stress surrounding cells have a negative impact on cell growth $(41,48,49)$.

These results supported that microstructures lent themselves in favour of cell growth without specific harmful effects on it and efficiently transported oxygen and nutrition to enclosed cells. However, further following of mitochondrial activity showed that it reached a plateau after the 21 st day, which means microparticles possibly suppressed cell growth (44).

\subsection{Spherical microtissue fabrication}

By the time the gradual aggregation of enclosed cells powerfully confirmed the presence of cell-cell interaction, which is crucial for the preservation of cell viability (24). These microparticles provided cellcell and cell-matrix interactions resulted in strong adhesive spherical microtissue with a more sophisticated microenvironment than the single cell (50). As it was shown in (Fig. 5I), the remaining spherical microtissue of L929 cells after microparticles digestion using collagenase supported the mentioned claim, making this procedure convenient to apply for spherical tissue formation to use in disease modeling (28), drug discovery (29), and tissue engineering purposes (30,31). Moreover, collagenase used to digest microparticles is naturally exists in the body tissues (51), so microparticles can be used as cell and drug delivery vehicles in vivo (35).

\section{Conclusions}

In this research, we have explored the feasibility of forming SF based cell-laden microparticles with a coaxial microfluidic device. Resultant microscopic images analyzed approved no evidence of agglomerations and non-uniformity in size and shape. Also, microparticles diameters controlled in uniform size. The quantitative analysis on cell viability shows cellular growth, indicating the shear stress imposed on cells during the microfluidic generation of microparticles, and the following gelation process does not cause apparent cell damage or death. Resultant images from enclosed cells confirmed that microparticles supported cell growth until the 15th day. Stability of cell-laden microparticles was related to the sufficient crosslinking among SF-Ph molecules through HRP-mediated crosslinking. The effective crosslinking method was validated by following up microparticles' microscopic images, showing the spherical formation and the lake of deformation or breakage during the process. Results illustrated high biocompatibility of the synthesis, microencapsulation process, cell proliferation, and successful spherical microtissue formation using SF derivative, showing this method's versatility for microparticles production. 


\section{Declarations}

\section{Ethics approval and consent to participate}

Not applicable

Consent for publication

Not applicable

\section{Availability of data and materials}

The datasets used and/or analyzed during the current study are available from the corresponding author on reasonable request.

\section{Competing interests}

The authors declare that they have no competing interests.

\section{Funding}

Not applicable

\section{Authors' contributions}

N. M and V. T. N have designed the experiments. E. B and M. $\mathrm{H}$ have performed synthesis and silk based microparticle fabrication. S. H and A. G performed MTT and degradation tests. M. Kh and M. J has led the project and wrote the paper. Z. A was the microfluidic device fabrication advisor. All authors have read and approved the final manuscript.

\section{Acknowledgements}

The authors gratefully acknowledge Iran University of Medical Sciences for the financial support of the project.

\section{Conflict of Interest}

I wish to confirm that there are no known conflicts of interest associated with this publication and there has been no significant financial support for this work that could have influenced its outcome.

I confirm that the manuscript has been read and approved by all named authors and that there are no other persons who satisfied the criteria for authorship but are not listed. I further confirm that the order of authors listed in the manuscript has been approved by all of authors.

I confirm that the authors have given due consideration to the protection of intellectual property associated with this work and that there are no impediments to publication, including the timing of 
publication, with respect to intellectual property. In so doing I confirm that we have followed the regulations of our institutions concerning intellectual property.

\section{References}

1. Zheng H \& Zuo B (2020) Functional silk fibroin hydrogels: preparation, properties and applications. Journal of Materials Chemistry $B$.

2. Brown J, Lu C-L, Coburn J, \& Kaplan DL (2015) Impact of silk biomaterial structure on proteolysis. Acta biomaterialia 11:212-221.

3. Gholipourmalekabadi M, et al. (2020) Silk fibroin for skin injury repair: where do things stand? Advanced drug delivery reviews 153:28-53.

4. Chung T-W, Chen W-P, Tai P-W, Lo H-Y, \& Wu T-Y (2020) Roles of Silk Fibroin on Characteristics of Hyaluronic Acid/Silk Fibroin Hydrogels for Tissue Engineering of Nucleus Pulposus. Materials 13(12):2750.

5. Talebian S, et al. (2019) Self-healing hydrogels: the next paradigm shift in tissue engineering? Advanced Science 6(16):1801664.

6. Thurber AE, Omenetto FG, \& Kaplan DL (2015) In vivo bioresponses to silk proteins. Biomaterials 71:145-157.

7. Hasturk O, Jordan KE, Choi J, \& Kaplan DL (2020) Enzymatically crosslinked silk and silk-gelatin hydrogels with tunable gelation kinetics, mechanical properties and bioactivity for cell culture and encapsulation. Biomaterials 232:119720.

8. Feng J, et al. (2020) Sustained release of bioactive IGF-1 from a silk fibroin microsphere-based injectable alginate hydrogel for the treatment of myocardial infarction. Journal of Materials Chemistry $B$.

9. Shimanovich U, et al. (2017) Silk micrococoons for protein stabilisation and molecular encapsulation. Nature communications 8(1):1-9.

10. Fathi A, et al. (2020) Fabrication of chitosan-polyvinyl alcohol and silk electrospun fiber seeded with differentiated keratinocyte for skin tissue regeneration in animal wound model. Journal of Biological Engineering 14(1):1-14.

11. Kasoju N \& Bora U (2012) Silk fibroin in tissue engineering. Advanced healthcare materials 1(4):393412.

12. Wang L, et al. (2018) Silk-graphene hybrid hydrogels with multiple cues to induce nerve cell behavior. ACS Biomaterials Science \& Engineering 5(2):613-622.

13. Chen D, et al. (2017) Orientational behaviors of silk fibroin hydrogels. Journal of Applied Polymer Science 134(32):45050.

14. Zhang F, et al. (2015) Excellent Cell Compatibility in Time Controlled Silk Fibroin Hydrogels. Materials Science Forum, (Trans Tech Publ), pp 407-411. 
15. Bae SB, Kim MH, \& Park WH (2020) Electrospinning and dual crosslinking of water-soluble silk fibroin modified with glycidyl methacrylate. Polymer Degradation and Stability 179:109304.

16. Bucciarelli A, et al. (2019) Preparation and statistical characterization of tunable porous sponge scaffolds using UV cross-linking of methacrylate-modified silk fibroin. ACS Biomaterials Science \& Engineering 5(12):6374-6388.

17. Chen S, et al. (2020) Construction of injectable silk fibroin/polydopamine hydrogel for treatment of spinal cord injury. Chemical Engineering Journal 399:125795.

18. Teramoto H (2017) Intermolecular crosslinking of silk fibroin by click chemistry. J. Silk Sci. Technol. Jpn 25:17-25.

19. Arabpour Z, et al. (2019) Design and characterization of biodegradable multi layered electrospun nanofibers for corneal tissue engineering applications. Journal of Biomedical Materials Research Part A 107(10):2340-2349.

20. McGill M, Coburn JM, Partlow BP, Mu X, \& Kaplan DL (2017) Molecular and macro-scale analysis of enzyme-crosslinked silk hydrogels for rational biomaterial design. Acta biomaterialia 63:76-84.

21. Sundarakrishnan A, et al. (2016) Phenol red-silk tyrosine cross-linked hydrogels. Acta biomaterialia 42:102-113.

22. Chimene D, Lennox KK, Kaunas RR, \& Gaharwar AK (2016) Advanced bioinks for 3D printing: a materials science perspective. Annals of biomedical engineering 44(6):2090-2102.

23. Acosta-Cuevas JM, et al. (2021) Generation of Photopolymerized Microparticles Based on PEGDA Using Microfluidic Devices. Part 1. Initial Gelation Time and Mechanical Properties of the Material. Micromachines 12(3):293.

24. Visscher LE, et al. (2017) Breast augmentation and reconstruction from a regenerative medicine point of view: state of the art and future perspectives. Tissue Engineering Part B: Reviews 23(3):281293.

25. Jiang W, Li M, Chen Z, \& Leong KW (2016) Cell-laden microfluidic microgels for tissue regeneration. Lab on a Chip 16(23):4482-4506.

26. Zhao X, et al. (2016) Injectable stem cell-laden photocrosslinkable microspheres fabricated using microfluidics for rapid generation of osteogenic tissue constructs. Advanced Functional Materials 26(17):2809-2819.

27. Siltanen C, et al. (2016) Microfluidic fabrication of bioactive microgels for rapid formation and enhanced differentiation of stem cell spheroids. Acta biomaterialia 34:125-132.

28. Nzou G, et al. (2018) Human cortex spheroid with a functional blood brain barrier for high-throughput neurotoxicity screening and disease modeling. Scientific reports 8(1):1-10.

29. Lee JM, et al. (2018) Generation of uniform-sized multicellular tumor spheroids using hydrogel microwells for advanced drug screening. Scientific reports 8(1):1-10.

30. Bauer M, et al. (2012) Adult cardiac progenitor cell aggregates exhibit survival benefit both in vitro and in vivo. PLoS One 7(11). 
31. Mekhileri N, et al. (2018) Automated 3D bioassembly of micro-tissues for biofabrication of hybrid tissue engineered constructs. Biofabrication 10(2):024103.

32. Blocki A, et al. (2015) Microcapsules engineered to support mesenchymal stem cell (MSC) survival and proliferation enable long-term retention of MSCs in infarcted myocardium. Biomaterials 53:1224.

33. Zhao S, et al. (2016) Bioengineering of injectable encapsulated aggregates of pluripotent stem cells for therapy of myocardial infarction. Nature communications 7:13306.

34. Ashida T, Sakai S, \& Taya M (2013) Competing two enzymatic reactions realizing one-step preparation of cell-enclosing duplex microcapsules. Biotechnology progress 29(6):1528-1534.

35. Khanmohammadi M, Sakai S, Ashida T, \& Taya M (2016) Production of hyaluronic-acid-based cellenclosing microparticles and microcapsules via enzymatic reaction using a microfluidic system. Journal of Applied Polymer Science 133(16).

36. Khanmohammadi M, Sakai S, \& Taya M (2017) Impact of immobilizing of low molecular weight hyaluronic acid within gelatin-based hydrogel through enzymatic reaction on behavior of enclosed endothelial cells. International journal of biological macromolecules 97:308-316.

37. Heichel DL \& Burke KA (2019) Dual-Mode Cross-Linking Enhances Adhesion of Silk Fibroin Hydrogels to Intestinal Tissue. ACS Biomaterials Science \& Engineering 5(7):3246-3259.

38. Khanmohammadi M, Sakai S, \& Taya M (2019) Characterization of encapsulated cells within hyaluronic acid and alginate microcapsules produced via horseradish peroxidase-catalyzed crosslinking. Journal of Biomaterials Science, Polymer Edition 30(4):295-307.

39. Choe G, Park J, Park H, \& Lee JY (2018) Hydrogel biomaterials for stem cell microencapsulation. Polymers 10(9):997.

40. Khanmohammadi M, Zolfagharzadeh V, Bagher Z, Soltani H, \& Ai J (2020) Cell encapsulation in coreshell microcapsules through coaxial electrospinning system and horseradish peroxidase-catalyzed crosslinking. Biomedical Physics \& Engineering Express 6(1):015022.

41. Li W, et al. (2018) Microfluidic fabrication of microparticles for biomedical applications. Chemical Society Reviews 47(15):5646-5683.

42. Schulz A, et al. (2019) Tyramine-conjugated alginate hydrogels as a platform for bioactive scaffolds. Journal of Biomedical Materials Research Part A 107(1):114-121.

43. Tomita K, et al. (2016) Cryopreservation of a small number of human sperm using enzymatically fabricated, hollow hyaluronan microcapsules handled by conventional ICSI procedures. Journal of assisted reproduction and genetics 33(4):501-511.

44. Sakai S, et al. (2009) Enzymatically fabricated and degradable microcapsules for production of multicellular spheroids with well-defined diameters of less than $150 \mu \mathrm{m}$. Biomaterials 30(30):59375942.

45. Rezvantalab S \& Moraveji MK (2019) Microfluidic assisted synthesis of PLGA drug delivery systems. RSC advances 9(4):2055-2072. 
46. Nemati S, et al. (2017) Alginate-gelatin encapsulation of human endothelial cells promoted angiogenesis in in vivo and in vitro milieu. Biotechnology and bioengineering 114(12):2920-2930.

47. Zafar B, Mottaghitalab F, Shahosseini Z, Negahdari B, \& Farokhi M (2020) Silk fibroin/alumina nanoparticle scaffold using for osteogenic differentiation of rabbit adipose-derived stem cells. Materialia 9:100518.

48. Bagheri S, et al. (2021) Control of cellular adhesiveness in hyaluronic acid-based hydrogel through varying degrees of phenol moiety cross-linking. Journal of Biomedical Materials Research Part $A$ 109(5):649-658.

49. Liu Y, Sakai S, \& Taya M (2013) Impact of the composition of alginate and gelatin derivatives in bioconjugated hydrogels on the fabrication of cell sheets and spherical tissues with living cell sheaths. Acta biomaterialia 9(5):6616-6623.

50. Ryu N-E, Lee S-H, \& Park H (2019) Spheroid culture system methods and applications for mesenchymal stem cells. Cells 8(12):1620.

51. Wilhelm SM, et al. (1986) Human fibroblast collagenase: glycosylation and tissue-specific levels of enzyme synthesis. Proceedings of the National Academy of Sciences 83(11):3756-3760.

\section{Figures}



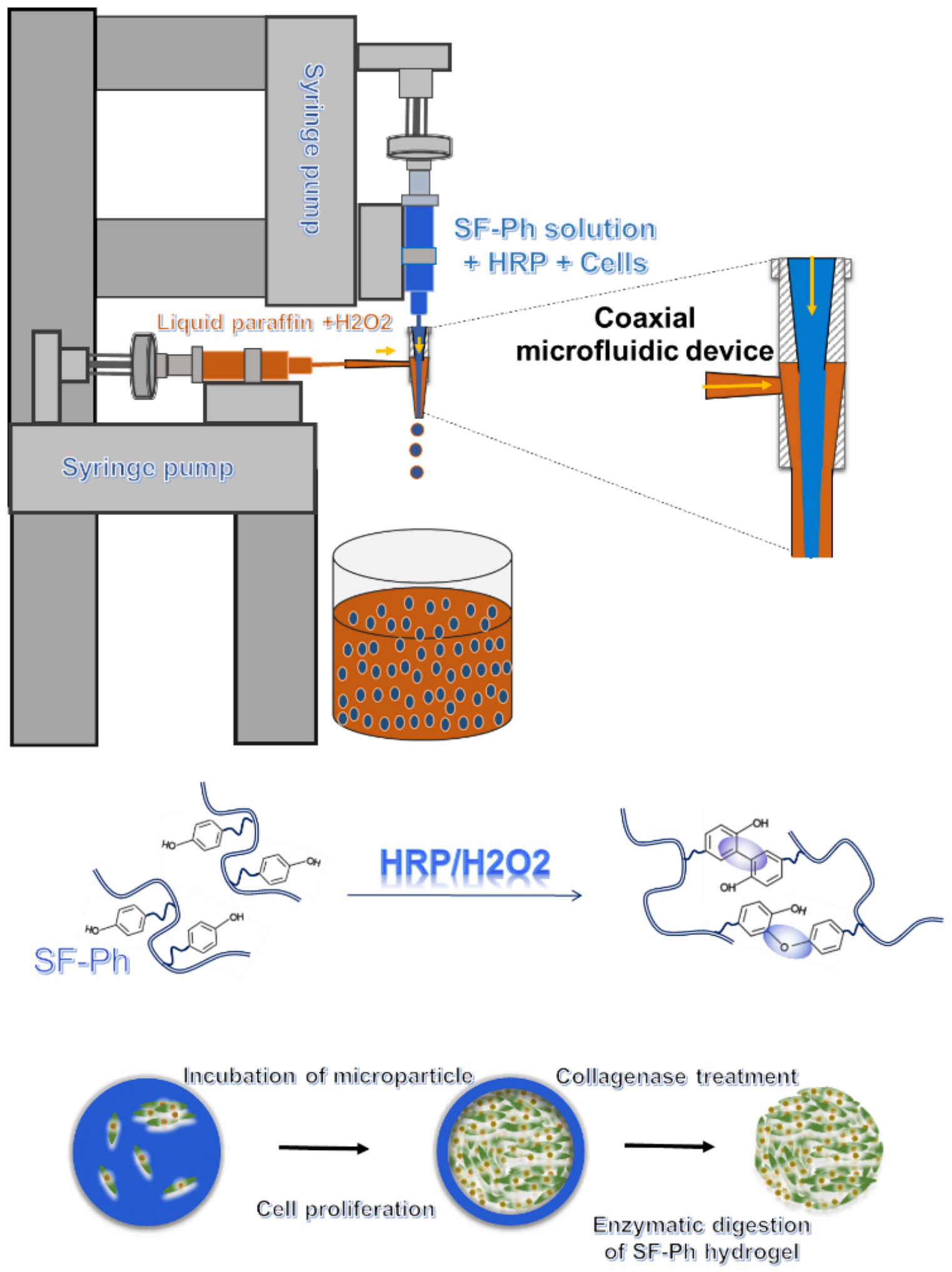

\section{Figure 1}

The production of cell enclosing silk derivative microsphere through the coaxial microfluidic device and horseradish (HRP) crosslinking reaction. The procedure of 1) silk fibroin extraction from Bombyx mori cocoons, 2) synthesis of SF-Ph 3) fabrication of the cell-laden microparti-cles, and 4) micro-tissues. 


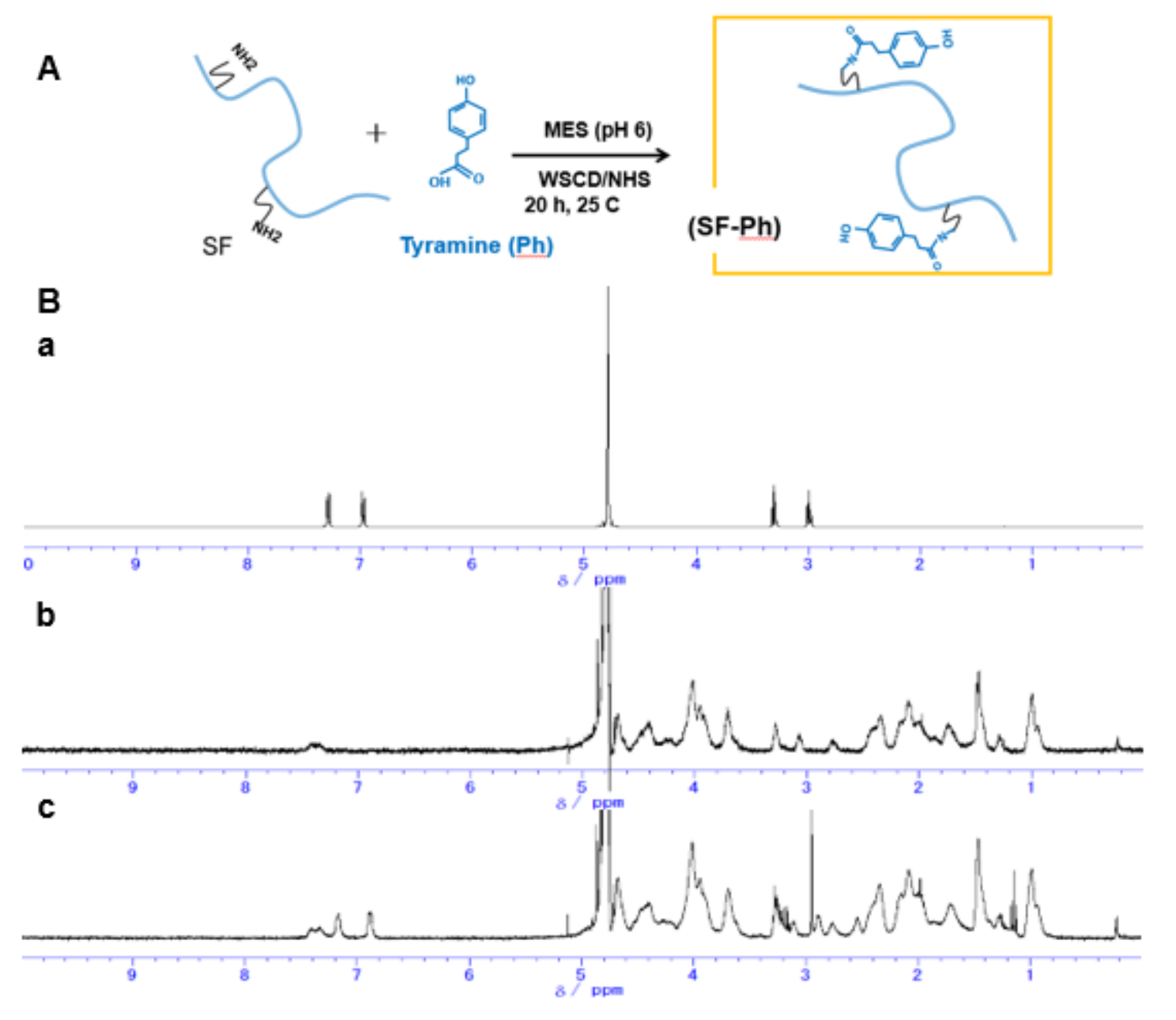

Figure 2

(A) The schematic illusion of SF-Ph synthesis using tyramine hydrochloride under WSCD and NHS catalyzers. (B) Results of 1H NMR for different materials: (a) tyramine hydrochloride, (b) unmodified SF, (c) modified SF (SF-Ph). 

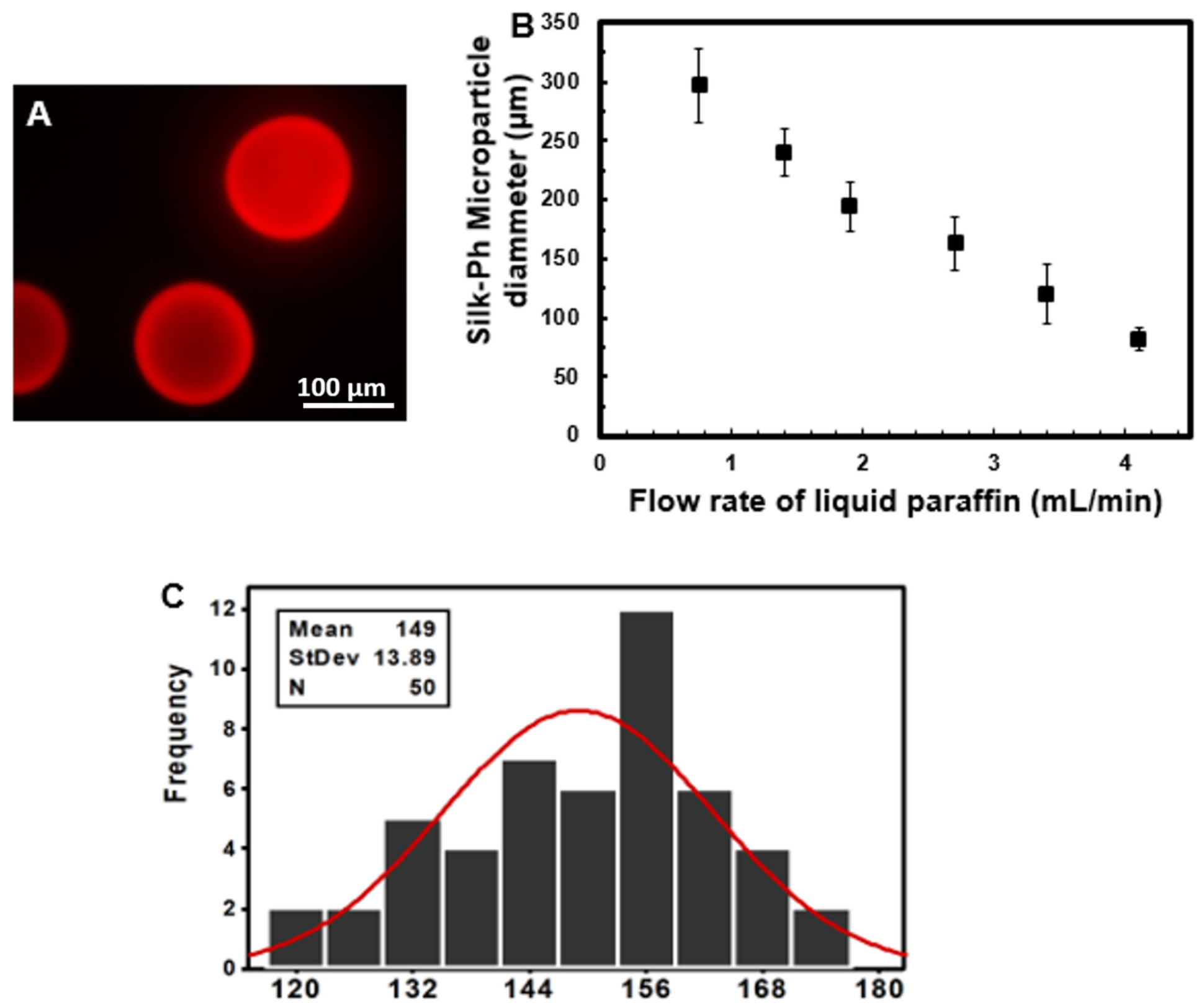

Figure 3

(A) Resultant microparticles stained with rhodamine, showing the feasibility of microparticles formation.

(B) The effect of liquid paraffin velocity containing H2O2 on SF-Ph microparticles diameter was investigated at the fixed flow velocity of the polymer solution ( $2 \mathrm{~mL} / \mathrm{min})$. (C) Diagram of microparticles size distribution at the specific flow rates of liquid paraffin $(2 \mathrm{~mL} / \mathrm{min})$ and SF-Ph solution $(75 \mu \mathrm{L} / \mathrm{min})$, considered for subsequent experiments. 

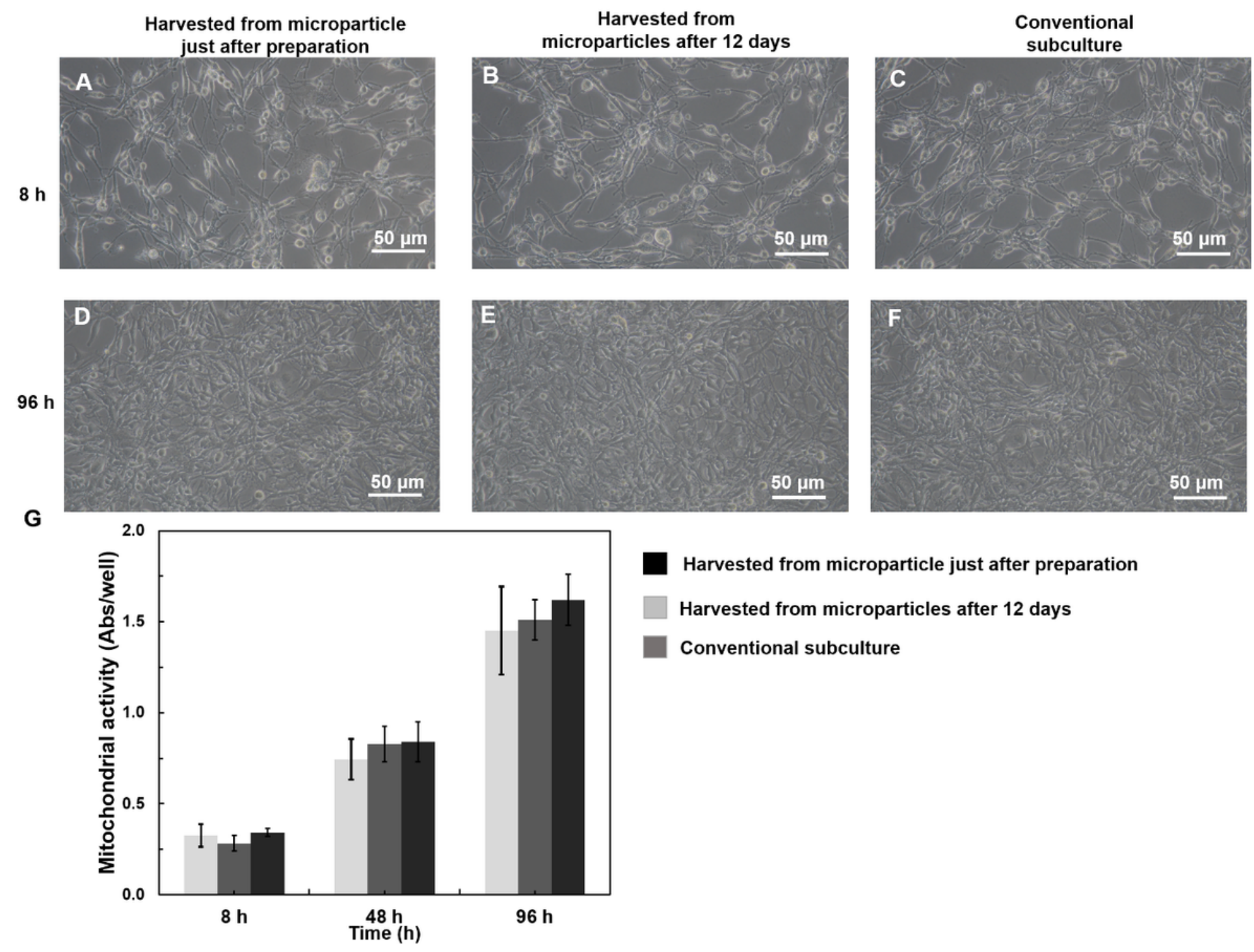

Harvested from microparticle just after preparation

Harvested from microparticles after 12 days

Conventional subculture

\section{Figure 4}

Microscopic images of cells cultured in an ordinary subculture (2 D), (A, B) Recovered cells from microparticles just and 12 days after microparticles preparation at day 1, (C) Control group (conventional subculture) at day 1. (D, E) Recovered cells from microparticles just and 12 days after microparticles preparation at $96 \mathrm{~h},(\mathrm{~F})$ Control group (conventional subculture) at $96 \mathrm{~h}$. (G) Diagram of mitochondrial activity of cells in a culture dishes for groups including recovered cells just and 12 days after microparticles preparation, and conventional subculture (Control) at different time points: 8, 48, and $96 \mathrm{~h}$. 
Day 1

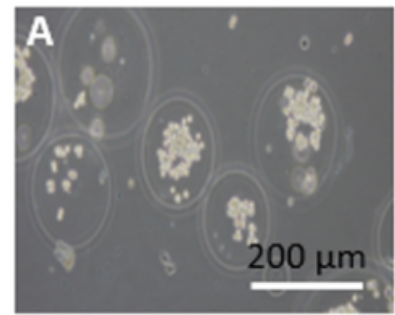

Day 9

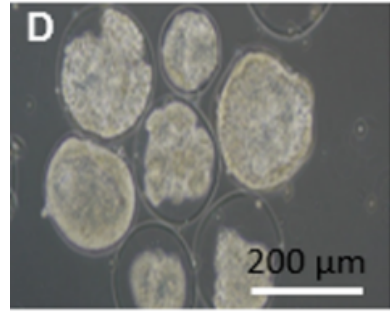

Day 3

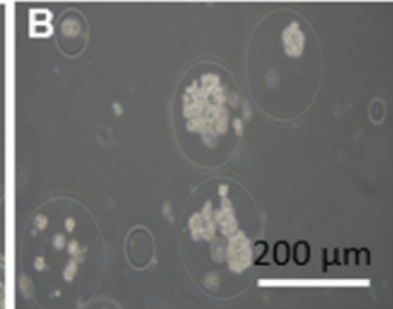

Day 12
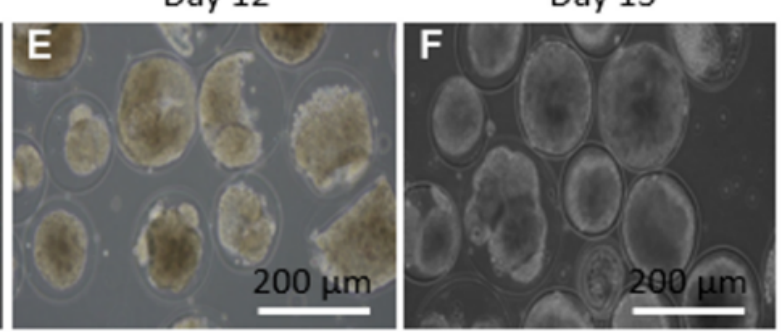

Day 6

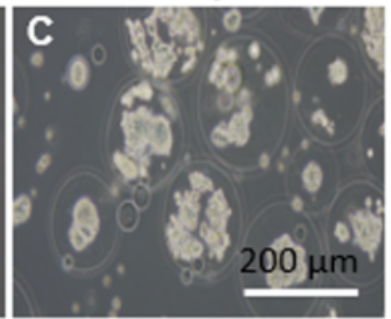

Day 15

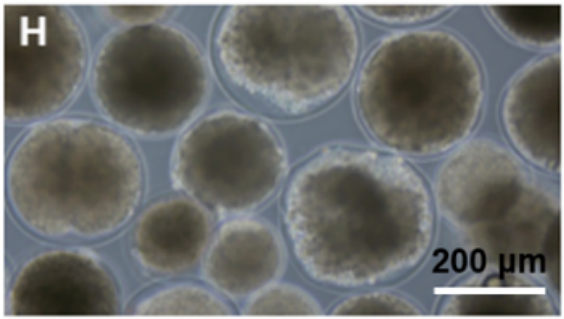

$\mathbf{G}_{0.7}$

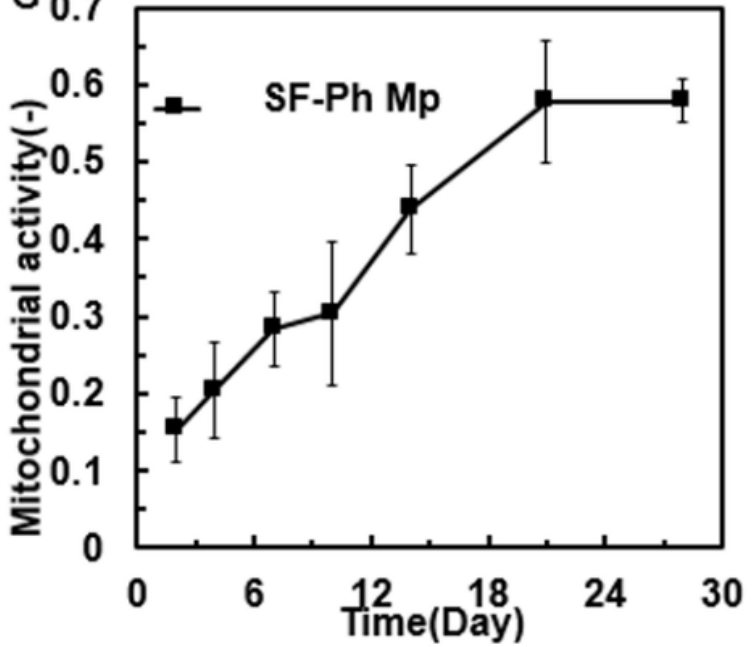

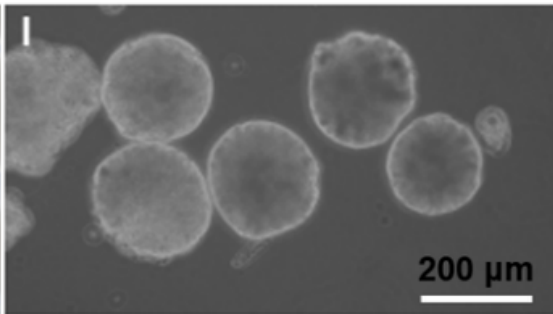

Figure 5

(A-F) Microscopic images from L929 enclosed cells growth in SF-Ph microparticles at different time points $(1,3,6,9,12$, and 15 days). (G) MTT assay diagram of L929 cells, showing cell proliferation in SF$\mathrm{Ph}$ microparticles. Microphotograph of cell-enclosing microparticles $\mathrm{H}$ ) before and (I) after degradation of SF-Ph microparticles by collagenase treatment. 